\title{
Correction to "The Orphan Receptor GPR17 Is Unresponsive to Uracil Nucleotides and Cysteinyl Leukotrienes"
}

In the above article [Simon K, Merten N, Schröder R, Hennen S, Preis P, Schmitt N-K, Peters L, Schrage R, Vermeiren C, Gillard M, Mohr K, Gomeza J, and Kostenis E (2017) Mol Pharmacol 91:518-532; DOI: https://doi.org/10.1124/mol.116.107904], there was an error in the published abstract. The bold portion of the abstract below is correct. The correct abstract will replace the incorrect abstract in all versions of the paper.

The authors regret this error and any inconvenience it may have caused.

\begin{abstract}
Pairing orphan G protein-coupled receptors (GPCRs) with their cognate endogenous ligands is expected to have a major impact on our understanding of GPCR biology. It follows that the reproducibility of orphan receptor ligand pairs should be of fundamental importance to guide meaningful investigations into the pharmacology and function of individual receptors. GPR17 is an orphan receptor characterized by some as a dualistic uracil nucleotide/cysteinyl leukotriene receptor and by others as inactive toward these stimuli altogether. Whereas regulation of central nervous system myelination by GPR17 is well established, verification of activity of its putative endogenous ligands has proven elusive so far. Herein we report that uracil nucleotides and cysteinyl leukotrienes do not activate human, mouse, or rat GPR17 in various cellular backgrounds, including primary cells, using eight distinct functional assay platforms based on labelfree pathway-unbiased biosensor technologies, as well as canonical second-messenger or biochemical assays. Appraisal of GPR17 activity can neither be accomplished with co-application of both ligand classes, nor with exogenous transfection of partner receptors (nucleotide P2Y12, cysteinyl-leukotriene CysLT1) to reconstitute the elusive pharmacology. Moreover, our study does not support the inhibition of GPR17 by the marketed antiplatelet drugs cangrelor and ticagrelor, previously suggested to antagonize GPR17. Whereas our data do not disagree with a role of GPR17 per se as an orchestrator of central nervous system functions, they challenge the utility of the proposed (ant)agonists as tools to imply direct contribution of GPR17 in complex biologic settings.
\end{abstract}

